\title{
Nanoindentation Size Effect Interpreted by the Dislocation Nucleation Mechanism
}

\author{
Xiaoming Liu, ${ }^{1}$, Zhanli Liu², Zuoqi Zhang ${ }^{3}$, Zhuo Zhuang ${ }^{2}$, and Yueguang Wei ${ }^{1}$ \\ ${ }^{1}$ LNM, Institute of Mechanics, Chinese Academy of Science, Beijing 100080, China \\ ${ }^{2}$ AML, Department of Engineering Mechanics, Tsinghua University, Beijing 100084, China \\ ${ }^{3}$ Institute of High Performance Computing, 138632, Singapore
}

\begin{abstract}
The instability criterion of atomic structure is used to investigate the nanoindentation size effect based on the dislocation nucleation mechanism. Both the dislocation nucleation location and the emission direction under indentation can be predicted. The nanoindentation size effect for hardness can be interpreted with a critical condition of the dislocation nucleation through adopting an Atomic Finite Element Method which is associated with the instability criterion. The present results agree well with the analytical solution based on the Rice model. The present results show that the nanoindentation size effect with the flat indenter can be expressed by using the dislocation nucleation concept. From dislocation nucleation point of view, the applied average force (hardness) required for dislocation emission is proportional to the inverse of square root of the width of the nano-indenter (contact zone size).
\end{abstract}

Keywords: Nanoindentation Size Effect, Dislocation Nucleation, Atomic Finite Element Method, Instability Criterion.

\section{INTRODUCTION}

Owing to the unique properties, researches on nanomaterials have attracted a great amount of attentions. As a primary measurement, nano-contact experiments such as nanoindentation, ${ }^{1,2}$ nano-bending ${ }^{3}$ and nanocompression $^{4-7}$ etc. have been widely applied to testing the mechanical properties of materials in small scale. Size effect of material behavior has been displayed. Previously, explanations on such phenomena are mostly from the continuum approachs, such as Nix, Gao,${ }^{8}$ Huang et al. ${ }^{2,9}$ and Wei et al. ${ }^{10,11}$ modeled size effects through adopting the strain gradient plasticity theories. The explanation based on the strain gradient plasticity is valid at the scale of several tens of micron where discrete dislocation events are ignored. Thus, it is still vague for the nanoindentation size effect at the nano-scale, which is very useful for manipulating atoms at experiment of nano-scale.

At the nano-scale, dislocation nucleation has significant influence on the incipient plastic behavior of materials compared with dislocation propagation behavior. Numerous nanoindentation experiments have revealed that the onset of discontinuous plastic deformation is associated with dislocation nucleation under high stress induced by

\footnotetext{
*Author to whom correspondence should be addressed.
}

indenter. The dislocation nucleation induces a sudden load-drop in the load-displacement curves, thus it plays a critical role in the plastic behavior of material at the nanoscale. A number of methods have been used for investigating the dislocation nucleation phenomena, such as, Li et al. ${ }^{12}$ Zhu et al. ${ }^{13}$ and Liu et al. ${ }^{14}$ have determined the homogeneously nucleated dislocation based on the elastic stability theory. Miller et al. ${ }^{15}$ have proposed a criterion based on the atomic-scale acoustic tensor. Their models highlight the need for nonlocal considerations in the development of a nucleation criterion. Xu et al. ${ }^{16,17}$ have also reported the dislocation nucleation on free surfaces and surface steps based on a general variational boundary integral formulation of the Peierls-Nabarro dislocation model. These criteria are mostly used for perfect crystals under deformation gradient or high order strains, and can not be employed for the evaluation of strengths in the cases of non-uniform deformations or bodies of inhomogeneous atom structures, such as nano-wires, nano-films etc. Umeno et al. ${ }^{18-20}$ have developed an instability criterion of an atomic structure based on the energy balance of whole system. This method can be applied to the evaluation of the strength of arbitrary structures and materials.

In the present research, dislocation nucleation criterion for arbitrary atomic system is firstly presented. Compared to model based on uniform deformations of crystal, ${ }^{12-14}$ 
this model has the advantage that it can be used to deal with complicated configurations in the relatively small atomic systems, where the continuum approach is known to break down. ${ }^{21}$ The present dislocation nucleation model is implemented into Atomic-scale finite element method $(\mathrm{AFEM})^{22}$ to study the nanoindentation size effect based on the critical condition for dislocation nucleation. The simulation result is also validated by comparing with the analytical result based on the Rice model. ${ }^{23}$

\section{SIMULATION METHOD}

\subsection{Dislocation Nucleation Criterion of Arbitrary Atomic System}

In general, the equilibrium configurations of any structures can be determined by the state of minimal energy. This is still valid for atomic structures. The atom sites at equilibrium state are determined by minimizing total energy of the system. The total energy $E$ of the structure under an arbitrary deformation can be expressed as:

$$
E=U-W
$$

where $U$ denotes the strain energy and $W$ is the work done by the external load.

For a system composting of $n$ atoms, the internal energy stored between atoms is denoted as:

$$
U=U(R)=U\left(r_{1}, r_{2}, \ldots, r_{n}\right)
$$

where $R=\left(r_{1}, r_{2}, \ldots, r_{n}\right)$ indicates the vector consisting of the coordinates of each atom.

Taylor's series expansion of the total energy in terms of infinitesimal deformation $\Delta R$ around the equilibrium state is given by

$$
\begin{aligned}
E(R+\Delta R)= & E(R)+\sum_{i=1}^{n} \frac{\partial E}{\partial r_{i}} \Delta r_{i} \\
& +\frac{1}{2} \sum_{i=1}^{n} \sum_{j}^{n} \frac{\partial^{2} E}{\partial r_{i} \partial r_{j}} \Delta r_{i} \Delta r_{j}+\cdots
\end{aligned}
$$

The state of minimal energy requires:

$$
\frac{\partial E}{\partial r_{i}}=0, \quad i=1,2, \ldots, n
$$

Substituting Eq. (3) into Eq. (4), the deviation of the total energy during the infinitesimal deformation, $\Delta E$, is obtained,

$$
\Delta E=E(R+\Delta R)-E(R)=\frac{1}{2} \Delta R^{T} A \Delta R
$$

where $A$ is a matrix whose components are as follows

$$
A_{i j}=\frac{\partial^{2} E}{\partial r_{i} \partial r_{j}}
$$

The structure can be stable only when the value of $\Delta E$ keeps positive for arbitrary infinitesimal deformation, $\Delta R$.

$$
\Delta E>0, \text { for arbitrary } \Delta R
$$

Unstable deformation takes place when the value is negative, namely, dislocation can be nucleated once the $\Delta E$ is negative.

Suppose $\eta_{1}, \eta_{2}, \ldots, \eta_{n},\left(\eta_{1}<\eta_{2}<\cdots<\eta_{n}\right)$ are the eigenvalues of the matrix $A$. Equation (7) cannot be valid once the minimum eigenvalue $\eta_{1}$ becomes zero. This criterion can be used to keep the positive of the symmetrical matrix $A_{i j}$.

$$
\eta_{1}=0
$$

Suppose eigenvector $p_{1}$ corresponding to eigenvalue $\eta_{1}$ is the direction of the arbitrary deformation $\Delta R$. For deformation displacement field at the specific direction by $p_{1}$, the incremental energy $\Delta E$ will always keep zero.

$$
\Delta E=\left(k p_{1}^{T}\right) A\left(k p_{1}\right)=\lambda_{1} k^{2}=0
$$

where $k$ denotes the amplitude of deformation on direction $p_{1}$. Equation (9) keeps for any value of $k$. Thus, Eq. (8) is the dislocation nucleation criterion and the dislocation emission direction can be predicted by the deformation mode $p_{1}$.

\subsection{Numerical Simulations}

Molecule dynamics (MD) method is widely used to simulate the mechanical properties of nano-systems. Recently, Atomic-scale Finite Element Method (AFEM) ${ }^{22}$ has been used for simulation of nano-tube systems ${ }^{24,25}$ by taking atoms as the finite element nodes. Compared to traditional MD method, AFEM is of the same accuracy, but takes less cost. For quasi-static processes of atomic systems, the AFEM is an effective and robust atomistic simulation method. Moreover, the dislocation nucleation criterion (Eq. (8)) can be easily implemented into the AFEM. Therefore, the AFEM instead of classical MD is used in the present simulations.

As shown in Figure 1, the Cartesian coordinates are selected so that $X$-axis is parallel to free surface. Atoms are fixed at the both sides and the bottom. The indenter is a rigid flat punch at width $2 a$ and infinite length (plane strain assumption). The tip is punched to the substrate with a total force of $F=2 p a$, where $p$ is the average pressure (hardness). The punch force $F$ gradually increases from zero to the value until dislocation criterion Eq. (8) is satisfied.

The interaction between atoms is characterized by using classical LJ potential:

$$
U=4 \varepsilon\left(\left(\frac{r_{0}}{r}\right)^{12}-\left(\frac{r_{0}}{r}\right)^{6}\right)
$$




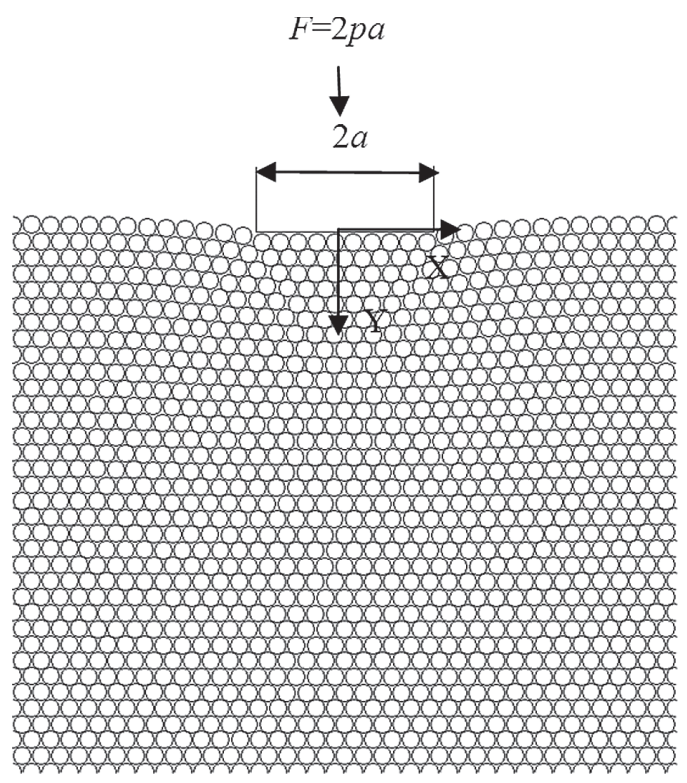

Fig. 1. Sketch of the simulation modal (plane strain case).

Table I. Normalized units used in simulations.

\begin{tabular}{lc}
\hline Name & Unit \\
\hline Length & $r_{0}$ \\
Energy & $\varepsilon$ \\
Stress & $\varepsilon / r_{0}^{3}$ \\
Force & Delivered by Publishing $\bar{c} r_{0}$ \\
\hline
\end{tabular}

where $\varepsilon$ and $r_{0}$ are the depth and radius of potential well, respectively. The normalized units used in simulations are listed in Table I. From these definitions, units of the other quantities (such as pressure, time, momentum etc.) are following directly. The equilibrium distance between atoms is taken as $a_{0}=1.1225 r_{0}$. The size of the indentation model is $150 a_{0} \times 75 \sqrt{3} a_{0}$, approximately 22000 atoms.

\section{RESULTS AND DISCUSSION}

For the case of a flat frictionless indenter pushed on the surface of the half-space, the distribution of normal traction within the contact region can be expressed by: ${ }^{26}$

$$
q(x)=\frac{2 p a}{\pi \sqrt{a^{2}-x^{2}}}
$$

Figure 2 shows the normal stress distributions along the contact interface with the indenter size $50 a_{o}$. The results from molecular simulation agree well with the analytical solution by Eq. (11).

Figure 3 shows the eigenvector $p_{1}$, corresponding to the instability criterion $\lambda_{1}=0$, which indicates the unstable deformation mode. For any deformation field at the specific direction denoted by the specific mode, the incremental energy $\Delta E$ will always keep zero. It can be seen from Figure 3(a) that the shear band originating from the edge

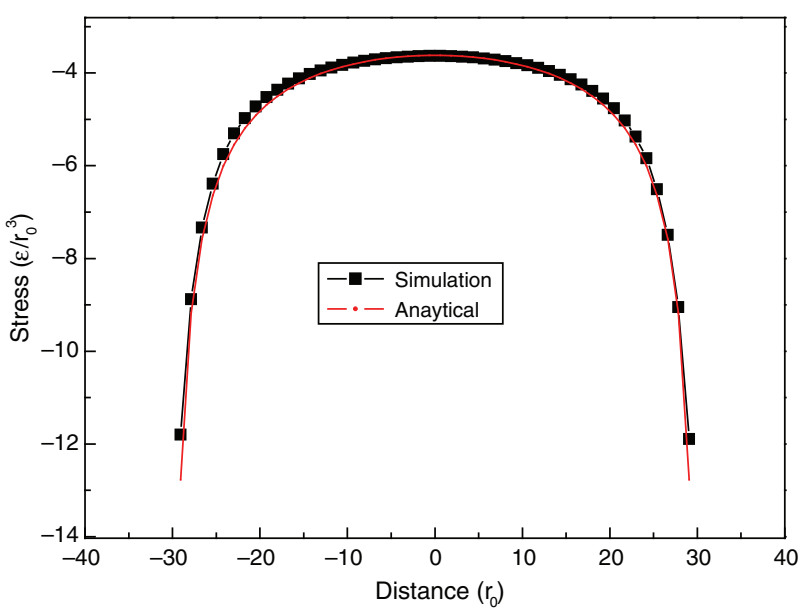

Fig. 2. Normal stress distributions on the contact interface.

of the indenter at the contact interface propagates down to the substrate. The relative slip directions are denoted by the arrows as shown in Figure 3. The contour plot of the unstable mode is shown in Figure 3(b). The dislocation nucleation site marked with black circle can be determined by the amplitude of the mode. The dislocation emission direction can be also successfully distinguished by the eigenvectors.

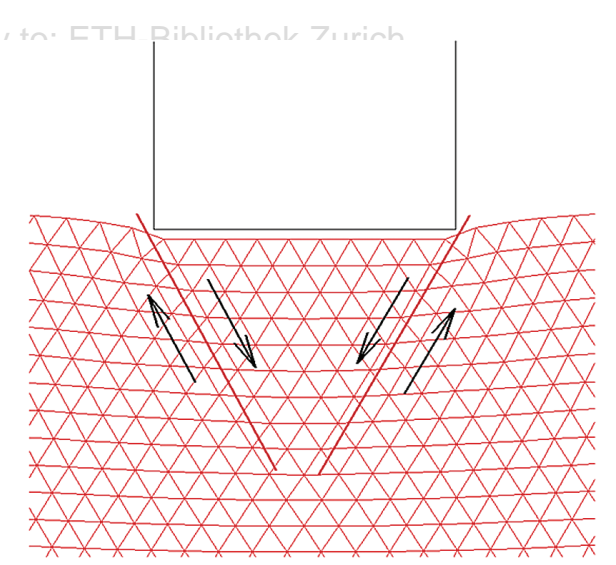

(a)

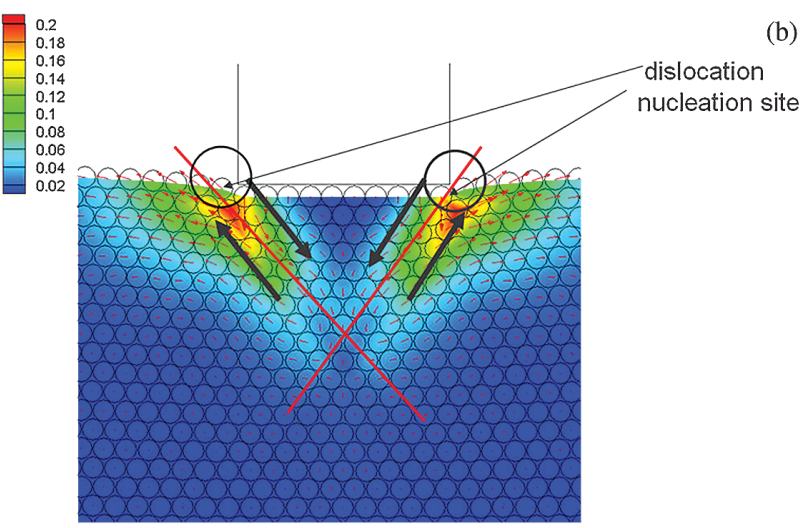

Fig. 3. Displacement of atoms at the unstable deformation mode evaluated by eigenvector. (a) Lattice distortion; (b) deformation contour. 
(a)

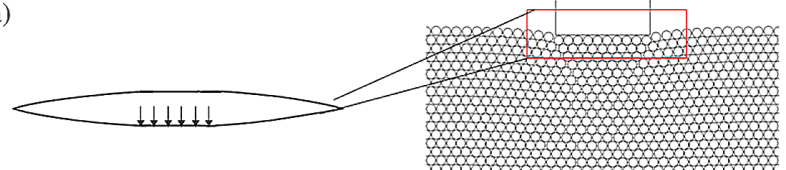

(b)

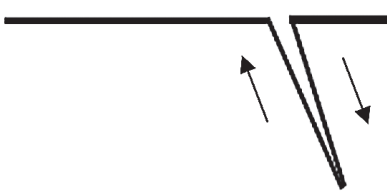

Fig. 4. Crack models used in the analysis (a) contact interface is modeled as part of the a large Mode I crack; (b) dislocation slip is modeled as a pure Mode II crack.

The singular stress field at the contact corner is solved by Nadai, ${ }^{27}$

$$
\sigma_{y}(r)=2 p \sqrt{\frac{a}{\pi}} \frac{1}{\sqrt{2 \pi r}}
$$

where $r$ is the distance from the contact edge. This is equivalent to the stress field of the mode I crack with the stress intensity factor $K_{\mathrm{I}}$ as shown in Figure 4(a),

$$
K_{\mathrm{I}}=2 p \sqrt{\frac{a}{\pi}}
$$

From Figure 4(b), it can be obtained that the dislocation nucleation corresponds to the mode II crack activation with the shear direction determined by the angle $\theta$ between the slip plane and the surface. The relationship between $K_{\text {I }}$ denoted by Eq. (13) and the effective stress intensity factor $K_{\mathrm{II}}$ of mode II was derived by Cotterell, ${ }^{28}$

$$
K_{\mathrm{II}}=C K_{\mathrm{I}}
$$

where $C$ is the constant depended on the angle between the slip plane and the surface, denoted by $\theta$.

$$
C=-\frac{3}{4}(\sin (\theta / 2)+\sin (3 \theta / 2))
$$

Based on the Rice model, ${ }^{23}$ the condition for dislocation nucleation is that the energy release rate in the slip plane direction is equal to the unstable stacking energy $\gamma_{u s}$,

$$
\frac{1-v}{2 \mu}\left(K_{\mathrm{II}}\right)^{2}=\gamma_{u s}
$$

where $\mu$ is the shear moduli.

Combining Eqs. (13)-(16), we can obtain the relationship between the average force (hardness) required for dislocation nucleation and the indenter size,

$$
p=Q / \sqrt{a}
$$

where $Q$ is defined by

$$
Q=\sqrt{\frac{\pi \mu \gamma_{u s}}{2(1-v) C^{2}}}
$$

From Eq. (18), $Q$ is a constant, depends on the material properties $\left(\mu, \gamma_{u s}\right)$ and dislocation nucleation direction $(\theta)$.

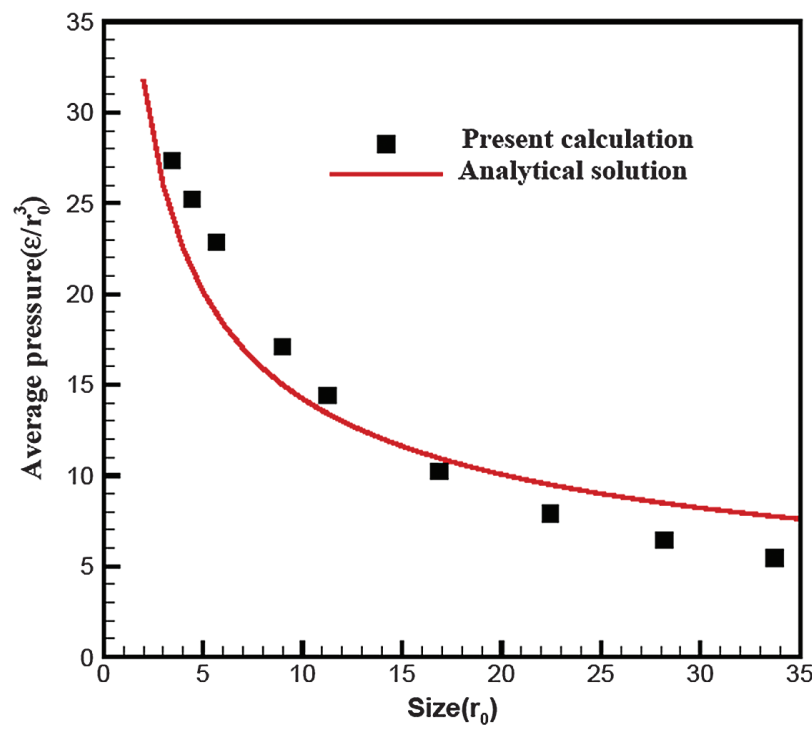

Fig. 5. Average pressure (hardness) as a function of the size of the indenter (contact zone size) based on the dislocation nucleation mechanism.

Figure 5 shows the relationship between the average pressures (hardness) required for dislocation nucleation and the size of the indenter (size of contact zone). The pressure decreases with increasing the contact zone size. The MD simulation results (denoted by dot in Fig. 5) agree well with the data deduced by analytical results using Eq. (17). The force required for incipient plastic behavior in nanoindentation experiment is proportional to the inverse of the square root of the width of the indenter (contact zone size). The lattice properties of copper $(\mathrm{Cu})$ by adopting L-J potential in Refs. [29, 30] are used in present work to validate calculation result. From the curve in Figure 5, the material constant $Q$ can be predicted, which is 45.03 in the unit of $\varepsilon / \sqrt{r_{0}^{5}}$ (it is $1.19 e 5 \mathrm{~J} / \sqrt{\mathrm{m}^{5}}$ in SI unit). And from simulation result, $\theta$ can be measured from Figures 3(a), (b), which is about $\pi / 3$. The shear modulus $\mu$ used here is about $110.5 \mathrm{GPa} .{ }^{29}$ The calculated unstable stacking fault energy is $\gamma_{u s}=64.21 \mathrm{~mJ} / \mathrm{m}^{2}$. It is in the range predicted by $3 \mathrm{D}$ molecular simulation result in Ref. [29] in which stable and unstable stacking fault energies are $\gamma_{s}=10.8 \mathrm{~mJ} / \mathrm{m}^{2}, \gamma_{u s}=220 \mathrm{~mJ} / \mathrm{m}^{2}$ respectively. The present results also agree with the analysis of dislocation nucleation under an idealized nanoindentation test based on Peierls-Nabarro dislocation model. ${ }^{31}$ It can be concluded that the loading capacity of crystal surface can be increased by increasing the indenter number while reducing the indenter size on the meantime.

\section{SUMMARY}

Dislocation nucleation criterions for uniform deformation have been widely studied in the past several years, and these works are mostly based on Cauchy-Born rule. Zhu et al. ${ }^{13}$ proposed low order theory and Miller et al. ${ }^{15}$ 
extended to the high order. The basic assumption for those previous works is that the deformation has to be uniform so that all the atoms' deformation can be represented by the deformation gradient (or high order deformation gradient) locally around the material point. However, this assumption can not be satisfied at the small scale in which affine deformation occurs hardly, such as nanowire (or nanofilm) system which has large part of surface atoms, flat nanoindentaion case which has the singularity at the contact edge, etc. Thus, continuum approach may not be effective in those cases. In our model, all the atom's freedoms are considered in the energy term to determine the instability mode instead of using homogenization by previous work. It is much more complete than homogenized model proposed before. With the aid of AFEM which makes not only convenient but also efficient to assemble the system stiffness matrix, it is highly possible to extend present method to other application such as nanowire or nanofilm systems.

In the present work, this model is used to investigate the dislocation nucleation mechanism under nanoindentation with a flat indenter. Dislocation nucleation site has been determined by using the instability criterion, while dislocation nucleation direction has been also successfully predicted by using the unstable deformation model. The nanoindentation size effect has been investigated based on the dislocation nucleation mechanism and through using the AFEM. The present results agree well with the analytical solution based on the Rice model. The results have also indicated that nanoindentation size effect is obvious based on the dislocation nucleation mechanism. The applied average force (hardness) required for dislocation emission is proportional to the inverse of the square root of the width of the indenter (or contact zone size).

Acknowledgment: This work was supported by the National Natural Science Foundation of China (Grants Nos. 10772096, 11021262, 10932011, 11142005, 11202214 and 91116003), and by National Basic Research Program of China through 2012CB937500. We thank Dr. B. Feng for helpful discussions.

\section{References}

1. K. W. McElhaney, J. J. Vlassak, and W. D. Nix, J. Mater. Res. 13, 1300 (1998).

2. S. Qu, Y. Huang, W. D. Nix, H. Jiang, F. Zhang, and K. C. Hwang, J. Mater. Res. 19, 3424 (2004).
3. B. Wu, A. Heidelberg, and J. J. Boland, Nat. Mater. 4, 525 (2005).

4. M. D. Uchic, D. M. Dimiduk, J. N. Florando, and W. D. Nix, 305, 986 (2004).

5. J. R. Greer, W. C. Oliver, and W. D. Nix, Acta Mater. 53, 1821 (2005).

6. Z. W. Shan, R. K. Mishra, S. A. S. Asif, O. L. Warren, and A. M. Minor, Nat. Mater. 7, 115 (2007).

7. Z. L. Liu, X. M. Liu, Z. Zhuang, and X. You, Scripta Mater. 60, 594 (2009).

8. W. D. Nix and H. Gao, Journal of the Mechanics and Physics of Solids 46, 411 (1998).

9. Y. Huang, S. Qu, K. C. Hwang, M. Li, and H. Gao, Int. J. Plast. 20, 753 (2004).

10. Y. G. Wei, S. Q. Shu, Y. Du, and C. Zhu, Int. J. Plast. 21, 2089 (2005).

11. Y. G. Wei, X. Z. Wang, and M. H. Zhao, J. Mater. Res. 19, 208 (2004).

12. J. Li, K. J. Van, Vliet, T. Zhu, S. Yip, and S. Suresh, Nature 418, 307 (2002).

13. T. Zhu, J. Li, J. K. Van Vliet, S. Ogata, S. Yip, and S. Suresh, Journal of the Mechanics and Physics of Solids 52, 691 (2004).

14. X. M. Liu, Z. L. Liu, X. You, J. F. Nie, and Z. Zhuang, Chinese Physics Letters 26, 026103 (2009).

15. R. E. Miller and D. Rodney, Journal of the Mechanics and Physics of Solids 56, 1203 (2008).

16. G. Xu and C. Zhang, Journal of the Mechanics and Physics of Solids 51, 1371 (2003).

17. C. Li and G. Xu, Philosophical Magazine 86, 2957 (2006).

18. S. V. Dmitriev, T. Kitamura, J. Li, Y. Umeno, K. Yashiro, and N. Yoshikawa, Acta Mater. 53, 1215 (2005).

19. Y. Umeno, T. Kitamura, and M. Tagawa, Mater. Sci. Eng. A 462, 450 (2007).

20. T. Kitamura, Y. Umeno, and R. Fushino, Mater. Sci. Eng. A 379, 229 (2004).

21. B. Luan and M. O. Robbins, Nature (London) 435, 929 (2005).

22. B. Liu, Y. Huang, H. Jiang, S. Qu, and K. C. Hwang, Computer Methods in Applied Mechanics and Engineering 193, 1849 (2004).

23. J. R. Rice, J. Mech. Phys. Solids 40, 239 (1992).

24. Z. Q. Zhang, B. Liu, Y. L. Chen, H. Jiang, K. C. Hwang, and Y. Huang, Nanotechnology 19, 395702 (2008).

25. Y. L. Chen, B. Liu, J. Wu, Y. Huang, H. Jiang, and K. C. Hwang, Journal of the Mechanics and Physics of Solids 56, 3224 (2008).

26. K. L. Johnson, Contact Mechanics, Cambridge University Press, Cambridge, United Kingdom (1987).

27. A. Nadai, Theory of Flow and Fracture of Solids, McGraw-Hill, New York, United States (1950), Vol. 1.

28. B. Cotterell and J. R. Rice, International Journal of Fracture 16, 155 (1980).

29. G. Ziegenhain, A. Hartmaier, and H. M. Urbassek, Journal of the Mechanics and Physics of Solids 57, 1514 (2009).

30. D. J. Quesnel, D. S. Rimai, and L. P. DeMejo, Phys. Rev. B 48, 6795 (1993).

31. C. Zhang and G. Xu, Mater. Sci. Eng. A 400, 471 (2005).

Received: 30 January 2012. Accepted: 4 February 2012. 